\title{
Use of NOACs in the Peri-Operative Management of Patients with Atrial Fibrillation: To Stop, Bridge or Continue?
}

\author{
Marco Proietti ${ }^{1,2,3}$ Deirdre A. Lane ${ }^{2}$ \\ ${ }^{1}$ IRCCS - Istituto di Ricerche Farmacologiche Mario Negri, \\ Milan, Italy \\ 2 Institute of Cardiovascular Sciences, University of Birmingham, \\ Sandwell and West Birmingham Hospitals NHS Trust, Birmingham, \\ United Kingdom \\ ${ }^{3}$ Department of Internal Medicine and Medical Specialties, Sapienza \\ University of Rome, Rome, Italy
}

Thromb Haemost 2018;118:1123-1126.

Over the last decade, the availability of non-vitamin K antagonist oral anticoagulants (NOACs) has significantly changed the daily clinical practice in managing thromboembolic risk in patients with atrial fibrillation (AF). ${ }^{1}$ Guidelines for AF management have established that NOACs are the preferred treatment for the majority of AF patients; ${ }^{2}$ however, recent data from 'real-life' registries clearly show that a significant proportion of patients are still treated with vitamin $\mathrm{K}$ antagonists. ${ }^{3-8}$ Nevertheless, the role of NOACs still seems to be debated in some specific circumstances, such as their role in the peri-operative management of patients with $\mathrm{AF}$, in relation to interruption or continuation of anticoagulation.

The management of oral anticoagulation (OAC) therapy in the peri-operative setting has been long debated. Despite the absence of solid evidence, bridging OAC therapy with unfractioned heparin or low molecular weight heparin (LMWH) was commonly suggested. ${ }^{9,10}$ In 2015, the 'Bridging Anticoagulation in Patients who Require Temporary Interruption of Warfarin Therapy for an Elective Invasive Procedure or Surgery' (BRIDGE) trial provided an answer to this important issue regarding warfarin, ${ }^{11}$ demonstrating that while patients undergoing bridging therapy with LMWH had a similar risk for thromboembolic complications, they were at significantly increased risk of major bleeding. ${ }^{11}$ Although there has been some clarification about peri-procedural management with warfarin, uncertainty still remains about peri-procedural NOAC management, due to the lack of solid data and limited clinical experience.

In the previous issue of Thrombosis and Haemostasis, Douketis and colleagues presented a sub-group analysis on peri-operative management and outcomes in AF patients treated with warfarin or edoxaban, derived from the "Effec-
Address for correspondence Marco Proietti, MD, IRCCS - Istituto di Ricerche Farmacologiche Mario Negri, Via Giuseppe La Masa 19, 20156, Milan, Italy (e-mail: marco.proietti@uniroma1.it).

tive Anticoagulation with Factor Xa Next Generation in Atrial Fibrillation-Thrombolysis in Myocardial Infarction 48" trial. ${ }^{12}$ Of the 21,105 patients originally enrolled in the trial, 7,193 (34.1\%) required surgery or an invasive procedure, and were equally randomized to warfarin, edoxaban high dose (60 or $30 \mathrm{mg}$ ) and edoxaban low dose ( 30 or $15 \mathrm{mg}$ ), with no major differences across the three groups. The most common procedures were cardiac, gastrointestinal endoscopy, dental and electrophysiological. Among those undergoing elective procedures, 3,116 (43.3\%) had their anticoagulation interrupted, defined as warfarin/edoxaban stopped for 4 to 10 days before the procedure, while 4,077 (56.7\%) were defined as 'anticoagulant continued', having stopped randomized treatment $\leq 3$ days (or not stopped at all) before the procedure. $^{12} \mathrm{~A}$ 30-day observation period was established to determine if there were differences in efficacy and safety between warfarin and the two doses of edoxaban in patients undergoing elective procedures, in patients with interrupted or continued anticoagulation treatment.

In the anticoagulant interrupted group, rates of stroke or systemic embolism at 30 days were $0.6,0.5$ and $0.9 \%$ for the warfarin, edoxaban high dose and edoxaban low dose groups, respectively ( $p=0.53$ for differences across the groups). Corresponding figures in the anticoagulation-continued group were $1.1,0.7$ and $0.9 \%$, with no significant between-group differences. Regarding the main safety outcome, major or clinically relevant non-major (CRNM) bleeding was reported in $3.9,4.2$ and $3.6 \%$ of patients receiving warfarin, edoxaban high dose and edoxaban low dose, respectively, for the anticoagulation interrupted group, with no significant differences across the three treatment groups. Among patients who continued anticoagulation, rates of major/CRNM bleeding in received

May 16, 2018

accepted

May 17, 2018 (c) 2018 Georg Thieme Verlag KG Stuttgart · New York
DOI https://doi.org/ 10.1055/s-0038-1661370. ISSN 0340-6245. 
Table 1 Evidence about peri-procedural management of NOACs from phase III trials

\begin{tabular}{|l|l|l|l|l|l|l|}
\hline Study & Year & NOAC & Patients & Procedures & Treatments & Results \\
\hline $\begin{array}{l}\text { Healey } \\
\text { et al }\end{array}$ & 2012 & Dabigatran & $4,591(25.3 \%)$ & - & $\begin{array}{l}\text { D110: 1,487 } \\
\text { D150: } 1,546 \\
\text { W: 1,558 }\end{array}$ & $\begin{array}{l}\text { Stroke/SE } \\
\text { D110 vs. W: RR, 1.05; 95\% Cl, 0.55-2.01 } \\
\text { D150 vs. W: RR, 1.01; 95\% Cl, 0.35-2.87 } \\
\text { Major Bleeding } \\
\text { D110 vs. W: RR, 0.83; 95\% Cl, 0.59-1.17 } \\
\text { D150 vs. W: RR, 1.09; 95\% Cl, 0.80-1.49 }\end{array}$ \\
\hline $\begin{array}{l}\text { Sherwood } \\
\text { et al }\end{array}$ & 2014 & Rivaroxaban & $4,692(33.0 \%)$ & 7,555 & $\begin{array}{l}\text { R: 2,165 } \\
\text { W: 2,527 }\end{array}$ & $\begin{array}{l}\text { Stroke/SE } \\
\text { R vs. W: HR, 0.74; } 95 \% \mathrm{Cl}, 0.36-1.50 \\
\text { Major Bleeding } \\
\text { R vs. W: HR, 1.26; 95\% Cl, 0.80-2.00 }\end{array}$ \\
\hline Garcia et al & 2014 & Apixaban & $5,439(29.9 \%)$ & 9,260 & $\begin{array}{l}\text { A: 2,701 } \\
\text { W: 2,738 }\end{array}$ & $\begin{array}{l}\text { Stroke/SE } \\
\text { A vs. W: OR, 0.60; 95\% Cl, 0.32-1.12 } \\
\text { Major Bleeding } \\
\text { A vs. W: OR, 0.85; 95\% Cl, 0.61-1.16 }\end{array}$ \\
\hline
\end{tabular}

Abbreviations: A, apixaban; Cl, confidence interval; D110, dabigatran 110 mg; D150, dabigatran 150 mg; HR, hazard ratio; NOAC, non-vitamin K antagonist oral anticoagulant; OR, odds ratio; $\mathrm{R}$, rivaroxaban; RR, relative risk; SE, systemic embolism; W, warfarin.

the three treatment arms $(p=0.17)$ were $8.5,7.9$ and $6.6 \%$, respectively. Mortality rates were similar across the three treatments whether or not anticoagulation was interrupted or continued. ${ }^{12}$ Results were comparable when analysed by type of procedure.

These results, strengthened by the sample size and the independently adjudicated outcomes, demonstrate that edoxaban can be safely used and managed in patients undergoing elective procedures but that safety appears to be improved if OAC is interrupted. These results, in particular comparing NOACs and warfarin in major adverse outcomes, reinforces and strengthens previous observations ( - Table $\mathbf{1}$ ) from sub-group analyses of other NOACs phase III trials, in patients undergoing elective procedures. ${ }^{13-15}$

The current European Society of Cardiology AF guidelines do not provide specific recommendations about the manage- ment of OAC therapy in patients undergoing cardiovascular procedures and interventions, but suggest that these interventions can be performed safely on continued OAC, and that on the basis of the BRIDGE trial discussed previously, bridging is not beneficial. ${ }^{2}$

More recently, the European Heart Rhythm Association released a 2018 update to its practical guide on the use of NOACs in AF patients, which discussed this issue in greater detail, ${ }^{16}$ proposing peri-operative management of OAC therapy as summarized in - Fig. 1. The main points emphasized by the practical guide relate to patients' baseline characteristics, in particular age and renal function, as well as the theoretical bleeding risk carried by the specific procedure, which needs to be taken into account when deciding whether to stop NOACs or not. Second, given the predictable effect and rapid clearance of NOACs, timely management of the planned

\section{Day -3 Day -2 Day -1 Day 0 Day +1 Day +2}

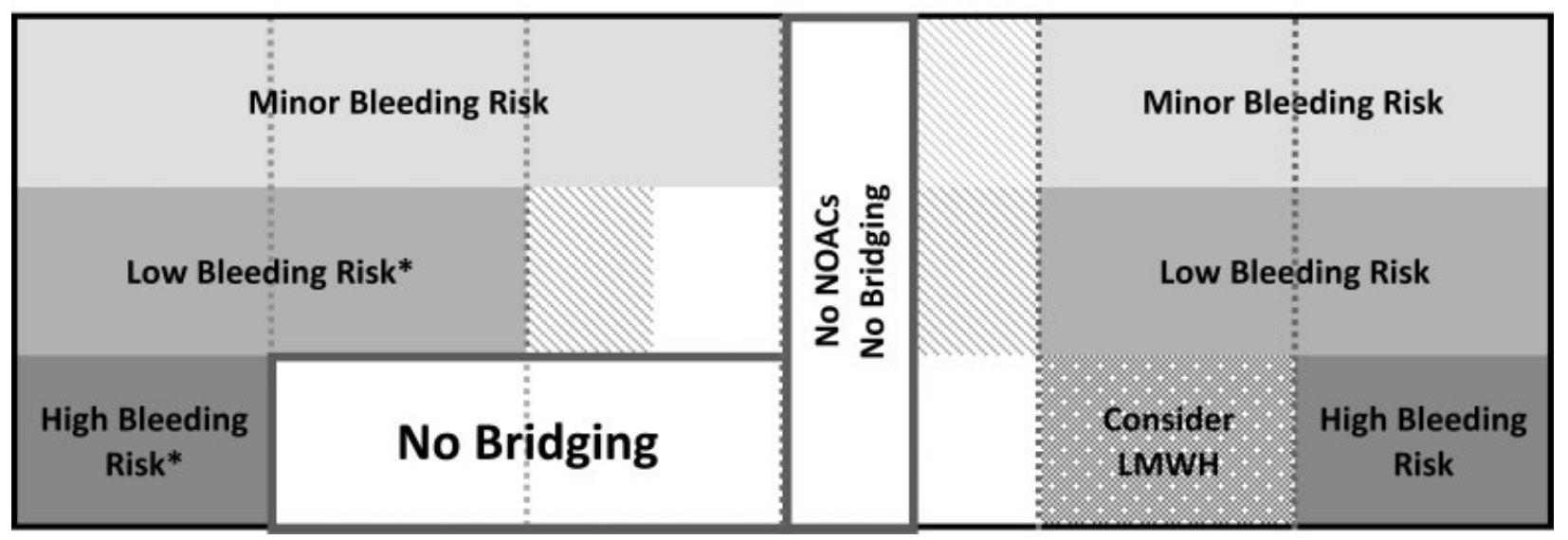

Fig. 1 Peri-operative management for NOACs in AF patients. OAC treatment can be continued or restarted according to bleeding risk of the procedure (solid blocks). In specific situations, OAC can stopped later or restarted earlier if bleeding risk is considered particularly low or the procedure reported an immediate and complete haemostasis (striped blocks). Under physicians' judgement, thromboprophylaxis with LMWH can be considered (dotted blocks). ${ }^{*}$ In patients taking dabigatran, drug must be stopped as much earlier as lower is the renal function. Abbreviations: AF, atrial fibrillation; LMWH, low molecular weight heparin; NOACs, non-vitamin $\mathrm{K}$ antagonist oral anticoagulants; OAC, oral anticoagulation. 
procedure can take advantage of the window between doses administration. ${ }^{16}$

Taking these aspects into consideration, in procedures with a minor bleeding risk (dental or eye procedures, diagnostic endoscopy and superficial surgery), all NOACs can be continued safely up to the day before the procedure. No NOACs are administered the morning of the procedure, and in those cases performed with no adverse events and with an immediate and complete haemostasis, NOACs can be restarted at the subsequent programmed dose, but not until 6 hours post-procedure. ${ }^{16}$

In those procedures with a low bleeding risk (biopsies, electrophysiological or procedures with cardiac implantable devices, non-coronary angiographies), stop NOACs up to 24 hours before the procedure, and recommence based on the same conditions as for minor bleeding risk procedures. ${ }^{16}$ For all those subjects undergoing procedures with a high risk of bleeding (complex endoscopies, major surgeries), the recommendation is cessation of NOACs at least 48 hours before surgery, without bridging. In these situations, NOACs can be restarted 48 hours after the procedures (or longer in specific situations) or post-procedure LMWH can be considered. ${ }^{16}$

In patients treated with dabigatran before the procedure, it is recommended to perform an accurate evaluation of renal function. In patients with a creatinine clearance $(\mathrm{CrCl})$ $\leq 80 \mathrm{~mL} / \mathrm{min}$, dabigatran should be stopped 12 hours earlier than in those with normal renal function $(>80 \mathrm{~mL} / \mathrm{min}$ ); 24 hours earlier in patients with $\mathrm{CrCl} \leq 50 \mathrm{~mL} / \mathrm{min}$; and 36 hours earlier in patients $\leq 30 \mathrm{~mL} / \mathrm{min}$. Similarly, in other specific situations in which the NOACs clearance could be prolonged, it is recommended to stop the treatment earlier. ${ }^{16}$

Another recent review about use of NOACs in surgical scenarios, proposed similar recommendations, underlining the importance of stratifying the procedure-predicted bleeding risk and of knowing the expected clearance time of the specific NOAC in the context of patient's age and co-morbidities. ${ }^{17}$

Notwithstanding the results provided by the NOAC phase III trials and expert recommendations, there is still a need for more specific peri-operative data from adequately powered randomized studies to provide definitive evidence, given the need to balance thromboembolic and bleeding risks in AF management. ${ }^{18}$ To address this, the "Perioperative Anticoagulant Use for Surgery Evaluation" study has been initiated (ClinicalTrials.gov NCT02228798), ${ }^{19}$ a prospective study with three parallel cohorts (dabigatran, rivaroxaban and apixaban), aiming to enrol 3,291 patients undergoing surgical procedures, to establish if the suggested NOAC and patient-specific interruption-resumption protocols are safe in the peri-operative management of $\mathrm{AF}$ patients. ${ }^{19}$ Results from this study will provide stronger evidence about the management of NOACs in this specific setting.

\section{Conclusion}

Despite the availability of data from sub-group analyses of the NOACs phase III trials and expert recommendations to reassure clinicians about the efficacy and safety of NOACs in the peri-operative management of AF patients (based on their predictability of anticoagulation effect, rapid clearance of effect), robust evidence from randomized controlled trials is still required to further clarify the risks associated with interruption or continuation of NOACs. Patient engagement, education and counselling are additional practical aspects to ensure safety while taking NOACs. ${ }^{20}$

\section{Conflict of Interest}

M.P. reports consulting activity for Boehringer Ingelheim outside the submitted work. D.A.L. reports educational grants from Bristol-Myers Squibb and Boehringer Ingelheim, speaker activity for Pfizer and consultant activity for Bristol-Myers Squibb, Bayer and Boehringer Ingelheim.

\section{References}

1 Lip G, Freedman B, De Caterina R, Potpara TS. Stroke prevention in atrial fibrillation: past, present and future. Comparing the guidelines and practical decision-making. Thromb Haemost 2017;117 (07):1230-1239

2 Kirchhof P, Benussi S, Kotecha D, et al; ESC Scientific Document Group. 2016 ESC Guidelines for the management of atrial fibrillation developed in collaboration with EACTS. Eur Heart J 2016;37 (38):2893-2962

3 Boriani G, Proietti M, Laroche C, et al; EORP-AF Long-Term General Registry Investigators. Contemporary stroke prevention strategies in 11096 European patients with atrial fibrillation: a report from the EURObservational Research Programme on Atrial Fibrillation (EORP-AF) Long-Term General Registry. Europace 2018;20(05):747-757

4 Li XS, Deitelzweig S, Keshishian A, et al. Effectiveness and safety of apixaban versus warfarin in non-valvular atrial fibrillation patients in "real-world" clinical practice. A propensity-matched analysis of 76,940 patients. Thromb Haemost 2017;117(06):1072-1082

5 Carmo J, Moscoso Costa F, Ferreira J, Mendes M. Dabigatran in real-world atrial fibrillation. Meta-analysis of observational comparison studies with vitamin $\mathrm{K}$ antagonists. Thromb Haemost 2016;116(04):754-763

6 Proietti M, Romanazzi I, Romiti GF, Farcomeni A, Lip GYH. Realworld use of apixaban for stroke prevention in atrial fibrillation: a systematic review and meta-analysis. Stroke 2018;49(01):98-106

7 Mazurek M, Huisman MV, Rothman KJ, et al; GLORIA-AF Investigators. Regional differences in antithrombotic treatment for atrial fibrillation: insights from the GLORIA-AF Phase II Registry. Thromb Haemost 2017;117(12):2376-2388

8 Hohnloser SH, Basic E, Hohmann C, Nabauer M. Effectiveness and safety of non-vitamin $\mathrm{k}$ oral anticoagulants in comparison to phenprocoumon: data from 61,000 patients with atrial fibrillation. Thromb Haemost 2018;118(03):526-538

9 Piazza G, Goldhaber SZ. Periprocedural management of the chronically anticoagulated patient: critical pathways for bridging therapy. Crit Pathw Cardiol 2003;2(02):96-103

10 Douketis JD, Spyropoulos AC, Spencer FA, et al. Perioperative management of antithrombotic therapy: Antithrombotic Therapy and Prevention of Thrombosis, 9th ed: American College of Chest Physicians Evidence-Based Clinical Practice Guidelines. Chest 2012;141(2, Suppl):e326S-e350S

11 Douketis JD, Spyropoulos AC, Kaatz S, et al; BRIDGE Investigators. Perioperative bridging anticoagulation in patients with atrial fibrillation. N Engl J Med 2015;373(09):823-833

12 Douketis JD, Murphy SA, Antman EM, et al. Peri-operative adverse outcomes in patients with atrial fibrillation taking warfarin or edoxaban: analysis of the ENGAGE AF-TIMI 48 trial. Thromb Haemost 2018;118(06):1001-1008 
13 Healey JS, Eikelboom J, Douketis J, et al; RE-LY Investigators. Periprocedural bleeding and thromboembolic events with dabigatran compared with warfarin: results from the Randomized Evaluation of Long-Term Anticoagulation Therapy (RE-LY) randomized trial. Circulation 2012;126(03):343-348

14 Sherwood MW, Douketis JD, Patel MR, et al; ROCKET AF Investigators. Outcomes of temporary interruption of rivaroxaban compared with warfarin in patients with nonvalvular atrial fibrillation: results from the rivaroxaban once daily, oral, direct factor Xa inhibition compared with vitamin $\mathrm{K}$ antagonism for prevention of stroke and embolism trial in atrial fibrillation (ROCKET AF). Circulation 2014; 129(18):1850-1859

15 Garcia D, Alexander JH, Wallentin L, et al. Management and clinical outcomes in patients treated with apixaban vs warfarin undergoing procedures. Blood 2014;124(25):3692-3698

16 Steffel J, Verhamme P, Potpara TS, et al; ESC Scientific Document Group. The 2018 European Heart Rhythm Association Practical Guide on the use of non-vitamin K antagonist oral anticoagulants in patients with atrial fibrillation. Eur Heart J 2018;39(16): 1330-1393

17 Verma A, Ha ACT, Rutka JT, Verma S. What surgeons should know about non-vitamin $\mathrm{K}$ oral anticoagulants: a review. JAMA Surg 2018. Doi: 10.1001/jamasurg.2018.0374

18 Nielsen PB, Skjøth F. A two-sided evaluation of benefit and harm from antithrombotic treatment in atrial fibrillation: balancing clinical application and statistical methodology. Thromb Haemost 2016;116(03):405-406

19 Douketis JD, Spyropoulos AC, Anderson JM, et al. The Perioperative Anticoagulant Use for Surgery Evaluation (PAUSE) study for patients on a direct oral anticoagulant who need an elective surgery or procedure: design and rationale. Thromb Haemost 2017;117(12):2415-2424

20 Loewen PS, Ji AT, Kapanen A, McClean A. Patient values and preferences for antithrombotic therapy in atrial fibrillation. A narrative systematic review. Thromb Haemost 2017;117(06): 1007-1022 